\title{
Anti-human immunodeficiency virus type 1 humoral immune response and highly active antiretroviral treatment
}

\author{
Vera Bongertz ${ }^{+}$, E Priscilla Ouverney, Saada Chequer Fernandez, Beatriz Grinsztejn*, \\ Valdilea Veloso*, José C Couto-Fernandez, José H Pilotto*, Mariza G Morgado
}

Laboratório de AIDS e Imunologia Molecular, Instituto Oswaldo Cruz-Fiocruz, Av. Brasil 4365, 21045-900 Rio de Janeiro, RJ, Brasil

*Instituto de Pesquisa Clínica Evandro Chagas-Fiocruz, Rio de Janeiro, RJ, Brasil

Highly active antiretroviral treatment (HAART) of human immunodeficiency type 1 (HIV-1) infection is very effective in controlling infection, but elimination of viral infection has not been achieved as yet, and upon treatment interruption an immediate rebound of viremia is observed. A combination of HAART with an immune stimulation might allow treatment interruption without this rebounding viremia, as the very low viremias observed with successful HAART may be insufficient to permit maintenance of a specific anti-HIV-1 immune response. The objective of this study was to compare the humoral immune response of individuals undergoing successful HAART ( $N F=$ no failure) with that of individuals with evidence of failure of therapy (FT) and to verify if the viremia peaks observed in individuals with therapy failure would act as a specific stimulus for the humoral anti-HIV-1 immune response. Antibodies binding to gp120 V3 genotype consensus peptides were more frequently observed for FT, mainly against peptides corresponding to sequences of genotypes prevalent in the Rio de Janeiro city area, B and F. HIV-1 neutralization of HIV-1 IIIB and of four primary isolates from Rio de Janeiro was less frequently observed for plasma from the NF than the FT group, but this difference was more expressive when plasma from individuals with detectable viremia were compared to that of individuals with undetectable viral loads in the year before sample collection. Although statistically significant differences were observed only in some specific comparisons, the study indicates that presence of detectable viremia may contribute to the maintenance of a specific anti-HIV-1 humoral immune response.

Key words: antibodies - seroreactivity - neutralization - antiretroviral treatment - treatment failure

Anti-HIV highly active antiretroviral treatment (HAART) reduces HIV in peripheral blood and reverts the characteristic immunodeficiency, and has benefited patients increasing individual survival at least 13-14 years (Vermund et al. 2006). However, no elimination of viral infection has been achieved, and the antiretroviral drugs will probably have to be taken throughout the life of the patient. Attempts to stimulate a potent specific anti-HIV-1 immune response to permit control of viral replication. After HAART has reduced viremia to undetectable levels, have so far been less than successful. During successful HAART, a decline in antibody response is generally observed (Fournier et al. 2002, Devito et al. 2006), with cases of seroreversion being described (Amor et al. 2006) and the extent of immune reconstitution in HAART treated individuals is controversial, apparently depending on the immune status of the individuals at the start of therapy (Cheonis et al. 2005). However, the benefits of HAART are evident even when treatment apparently fails, leading neither to a complete control of viremia nor to a complete reconstitution in CD4 T lymphocytes (Brígido et al. 2004, Kovacs et al. 2005).

Financial support: PAPES III - Fiocruz

${ }^{+}$Corresponding author: bongertz@ioc.fiocruz.br

Received 28 March 2007

Accepted 5 October 2007
During successful anti-HIV therapy, a reduction in virus specific cellular immunity occurs (Dalod et al. 1998), possibly due to inefficient CD8 T lymphocyte stimulation at the consequent very low antigenic concentrations (Bucy et al. 2001). It is also thought that the low level of HIV replication during successful HAART may prevent induction of a high titer antibody response (Popovic et al. 2005, Bailey et al. 2006), and, although sporadic cases of a high autologous neutralizing antibody (NAb) have been reported, the NAb response during HAART is poorly understood (Kimura et al. 2002, Dreyer et al. 1999). Titers of "binding antibodies" (bAb = antibodies detectable through their in vitro binding to synthetic proteins or peptides) have been shown to decrease during successful HAART (Morris et al. 2001, Bailey et al. 2006). However, generation of specific anti-HIV antibodies in absence of detectable viremia has been described, indicative of retention of HIV antigen over long periods (Kim et al. 2001, Popovic et al. 2005) and restoration of normal B cell functions during HAART (Morris et al. 1998, David et al. 1999). Nevertheless, there are indications that the moment of HAART initiation defines the consequential restoration in the immune response (Mocroft et al. 2003, Manzardo et al. 2007, Chehimi et al. 2007): the extent of immune response alterations caused in HIV infection will define restoration, but the quantitative immune stimulation induced by HIV will also be important for the extent of specific antiHIV response detectable during or after successful antiretroviral therapy. 
Intermittent structured treatment interruptions may stimulate antiviral immunity through an autoimmunization process (Jacobson et al. 2006), although this process seems to be controversial (Ioannidis et al. 2000). However, a stimulation of the CD8 T lymphocyte antinef response has been shown to occur (Benito et al. 2006). Also, some studies indicate that occasions of viral rebound or "blips" may benefit patients by increasing their specific immune response (Kimura et al. 2002, Sullivan et al. 2003, Karlsson et al. 2004, Macias et al. 2005), including the titers of bAb (Binley et al. 2000, Killian et al. 2006) and the NAb response (Binley et al. 2000, Montefiori et al. 2001, Kimura et al. 2002, Wang et al. 2002).

It is known that, depending on the drug treatment of the transmitting individuals, up to $20 \%$ of new patients were infected with strains that were already drug resistant (UK Collaborative Group on HIV Drug Resistance 2007). Resistance data are available for some countries, with rates increasing worldwide since 1997. Despite the large use of HAART-based regimens in the treatment of HIV/AIDS patients in Brazil, the rate of primary HIV drug resistance is very low when compared with that of developed countries (Soares et al. 2004).

The present study was carried out in an attempt to evaluate if an effective autoimmunization occurs with a stimulation of anti-HIV-1 humoral immune response in individuals who display peaks of viral load resulting from therapy failure, whether induced by lack of adherence to therapy schedules or by the appearance of drug resistant viral quasispecies. The drug-resistance mutations were evaluated in all patients of this study under treatment with HAART, through the genotyping of the HIV pol gene (Eyer-Silva et al. 2006).

\section{SUBJECTS, MATERIALS AND METHODS}

Plasma Samples - HIV-1 infected individuals followed at the Evandro Chagas Clinical Research Institute of Fiocruz between april 2003 and october 2004 were invited to participate in this study, and a total of 97 agreed in participating, after signing the informed consent form (project approved by the Fiocruz Ethical Committee n. 172/02). Results obtained from individuals selected to participate, but who did not meet the criteria defined for FT (failure of therapy), NF (no failure) or NT (no therapy) were excluded from the data shown. All patients had at least 4 medical visits, with lymphocyte typing (FACScan, Becton-Dickinson, San José, CA) and viral load determinations (nucleic acid sequence-based amplification NASBA, NucliSens, Organon Teknika, Boxtel, Netherlands). A total of 26 patients had been for at least one year in successful therapy, as indicated by decreasing viral loads and stable or increasing concentrations of CD4 lymphocytes in peripheral blood (group NF), and had no record of any therapy failure events or therapy interruptions. A group of 31 individuals in therapy with episodes of failure of therapy (FT), defined as patients experiencing virologic failure (sudden rises of HIV RNA to more than 5,000 copies $\mathrm{RNA} / \mathrm{ml}$, caused either by lack of adherence and/or viral resistance to antiretrovirals), called group FT, was included. As an outside control group, a total of 40 antiretroviral naive individuals (NT) were included. Table indicates mean values for information collected for the different groups. All patients were followed according to the Brazilian Ministry of Health HIV/ AIDS guidelines, receiving free antiretroviral therapy when numbers of circulating CD4 lymphocytes dropped below $350 / \mathrm{mm}^{3}$, viral loads were greater than 30,000 copies HIV-1 RNA/ml in peripheral blood or with viral loads increasing by more than one log in three months. Accordingly, all individuals receiving HAART agreed to collect four viral load and CD4 lymphocyte determinations per year and for evaluation of drug resistance mutation profiles.

Seroreactivity - Several custom made synthetic peptides (Chiron Mimotopes, San Diego, CA, USA) were

TABLE

Comparative statistical analyses of immunological status (\# CD4/mm3), viral load (VL, \# RNA copies/ml), time since HIV-1 serodiagnosis time post infection [p.i.] in months (m) and years (y) of antiretroviral treatment (ARV). Up to 1998, ARV used were one or two nucleoside reverse transcriptase inhibitors; after 1998 initiation of different protocols of three or more antiretroviral drugs with different mechanisms of action (HAART) was used. Samples were divided into groups of patients with failure of treatment (FT), success of treatment / no failure (NF) and treatment naive (NT), and results compared statistically by Students t-test

\begin{tabular}{lllll}
\hline & & FT & NF & NT \\
\hline \# CD4 & $\mathrm{n}$ & 26 & 31 & 39 \\
& Mean \pm SD & $243.6 \pm 243$ & $631 \pm 264$ & $586 \pm 279$ \\
VL & Range & $14-1028$ & $160-1284$ & $51-1559$ \\
& $\mathrm{n}$ & 26 & 31 & 40 \\
Time p.i. (m) & Median (log) & 4.20 & $<2$ & 4,24 \\
& Range & $79-750.000$ & $<80-27.000$ & $79-320.000$ \\
& Mean \pm SD & 26 & 30 & 40 \\
ARV (y) & Range & $100 \pm 6.6$ & $53 \pm 35$ & $38 \pm 29$ \\
& n & $60-168$ & $12-156$ & $1-108$ \\
& Mean \pm SD & 26 & 31 & \\
& Range & $7.48 \pm 2.1$ & $2.88 \pm 1.9$ & $1-11$ \\
\hline
\end{tabular}

$\mathrm{SD}=$ standard deviation. 
used: a) corresponding to the consensus sequences of the gp120 V3 loop of HIV-1 subtypes: A (KSVHIGPGQAFYAT), B (NTRKSIHIGPGRAFY), Brazilian Bbr (NTRKSIHMGWGRAFY), C (KSIRIGPGQTFYAT), D (RQRTHIGPGQALYTT), E (RTSITIGPGQVFFYR), F (RKSIHLGPQAFYTT), and of a representative Brazilian $\mathrm{F}$ isolate (FBR $=$ NTRKSIPLGPGRAFY), and $\mathrm{b}$ ) to the immunodominant epitope in gp41 of the HIV-1 isolate MN (ID = GFWGCSGKLICTTTVPWNAS). Duplicates of sequentially diluted (1:100 at a first step, followed by serial dilutions using factor 2) heat inactivated plasma were incubated $\left(1 \mathrm{~h} / 37^{\circ} \mathrm{C}\right.$ followed by an overnight incubation at $4^{\circ} \mathrm{C}$ ) with different biotinylated synthetic peptides $(5 \mathrm{mg} /$ well) bound to multiwell plates (Maxisorb, Nunc, Roskilde, Denmark) precoated with streptavidin $(10 \mathrm{mg} /$ well). Specific reactivity was assessed (including two 8M urea washes after antibody incubation according to Casseb et al. 2002) by peroxidase-conjugated anti-human-IgG binding and peroxide/ tetrametilbenzidine revelation. Reactivity was assessed as dilution titer reaching at least twice the mean binding of the duplicate control plasma pools (obtained from local HIV negative blood donors) included in each assay plate. Absorbency was measured at $450 \mathrm{~nm}$. For some of the samples tested in duplicate, reactivity with the peptides was discordant even after repetition, probably indicating low affinity binding partially removed by the $8 \mathrm{M}$ urea washes. These results were considered doubtful and not included in this report, leading to differences in numbers of samples tested with the different synthetic peptides (Bongertz et al. 2003).

Neutralizing Antibodies - Individual heat inactivated plasma from the different groups were tested as to their capacity to neutralize the reference HIV-1 isolate IIIB (kindly donated by Dr EM Fenyoe, Lund University, Sweden), adapted to replication in lymphocytic cells lines expressing CD4. For use in this study, viral stocks from two replication cycles in pre-activated normal human peripheral blood mononuclear cells (PHA-PBMC NHu, mixture of at least two donors) were pooled. First to third passage viral stocks of the primary HIV-1 isolates FT015 (subtype F, in this report called FT15), FT089 (subtype B, in this report called FT89), and third to fifth passage of 95BRRJ021 (subtype F, in this report called RJ21) and 95BRRJ020 (subtype B, in this report called RJ20) were also included (Bongertz et al. 2000). At least three 3-fold plasma dilutions, using 1:10 as the first step, were used.

Neutralization of the HIV-1 isolates was tested using PHA-PBMC NHu $\left(10^{5}\right.$ cells/well), using a multiplicity of infection (MOI) of 0.001-0.005 (10-50 infective units per well), according to the WHO-UNAIDS Guidelines (WHO-UNAIDS 2002). Quantification of the HIV-1 p24 antigen (HIV-1 p24 ELISA - Zeptometrix Corporation, Buffalo, NY) was carried out on the seventh day of cell culture. Positive control wells containing virus and cells (without human serum), assay wells containing virus, cells and serially diluted plasma, negative control wells (containing virus, cells and normal human serum), back- ground values (wells containing only cells) and wells used for determining the tissue culture infective dose $50 \%$ $\left(\mathrm{TCID}_{50 \%}\right)$ of the virus isolate were prepared simultaneously (Bongertz et al. 2001). Approximate plasma concentrations needed for neutralizing $90 \%$ and $50 \%$ of the viral input $(90 \%$ or $50 \%$ neutralization levels, respectively) were derived from linear regression curves (MicroCal Origin Software Inc, Northampton, MA, USA) or directly from the neutralization curve.

HIV-1 subtyping and drug resistance genotyping Genomic DNA was extracted from $200 \mathrm{ml}$ of whole blood from all 97 individuals included in this study using the QIAamp DNA kit (Qiagen Inc, Chatsworth, CA, USA), according to the manufacturer's protocol. The HIV-1 genetic subtype determination was performed analyzing the envelope (env) $\mathrm{C} 2-\mathrm{V} 3$ region of gp 120 , amplified by nested PCR as previously described (Guimaraes et al. 2002). The presence of drug resistance mutations to nucleoside reverse transcriptase inhibitors (NRTI), non-nucleoside RTI (NNRTI) and protease inhibitors (PI) was performed targeting two genomic regions of the polimerase (pol) gene, amplified by nested PCR, covering the whole protease (codons 1-99) and the first 235 codons of RT as described elsewhere (EyerSilva et al. 2006). All PCR products were purified using the Qiagen PCR purification kit (Qiagen) according to the manufacturer's protocol, sequenced using the ABI PRISM Big Dye Terminator Cycle Sequencing Ready Reaction Kit v.3.1 and analyzed in a ABI 3100 automate sequencer (Applied Biosystem, Foster City, CA, USA).

Resistance mutations and phylogenetic analyses Sequence chromatograms were aligned in PC/Windows using SeqMan software (DNAStar, Madison, WI) and manually edited. Edited sequences were then aligned to reference sequences representative of all HIV-1 subtypes obtained from the Los Alamos database (http://hivweb.lanl.gov) in ClustalW (Thompson et al. 1994). Aligned sequences from env and pol genes were subjected to phylogenetic inference through the neighborjoining method and Kimura 2-parameter model of the MEGA 2.1 package (Kumar et al. 2001) for assignment of HIV-1 subtype. The genotypic interpretation of antiretroviral drug resistant mutations in PR and RT was carried out by electronic submission to the Stanford database (http://hivdb.stanford.edu). Distinction of primary and secondary HIV-1 drug resistance mutations was based on the International AIDS Society guidelines (htpp:// iausa.org.us).

Statistical analyses - For evaluation of the results obtained the Instat software (Graph Instat tm, 19901994, San Diego, CA, USA) was employed, using the ANOVA non-parametric test, unpaired $t$-student test for comparison between ages, CD4 T cell counts, and viral loads; and contingency table (chi-square or exact Fisher test depending on sample numbers) for categorical variables. Linear regression was plotted using the Micro Cal Origin software (micro Cal Origin, Northampton, MA, USA, 1991-1992). 


\section{RESULTS}

Plasma Samples - All groups had a slightly higher participation of males and the majority indicated that infection occurred by sexual exposure, while ages varied from 18 to 60 . However, no statistically significant differences between groups were detected (data not shown). A summary of comparative CD4 lymphocyte numbers and viral loads at sample collection is shown in Table, indicating significantly lower counts of CD4 T lymphocytes (in comparison to the successfully treated and to the non-treated groups, $\mathrm{p}<0.0001)$ and higher viral loads in the FT group (in comparison to the successfully treated group $\mathrm{p}=0.0002$ and with the untreated control group $p=0.0008)$. Individuals had been infected for one to 12 years. In the successfully treated NF $(\mathrm{p}<$ $0.0001)$ and the NT control group $(\mathrm{p}<0.0001)$ a significantly higher number of individuals with periods since positive anti-HIV-1 serology of less than two years was identified in comparison to the FT group. Duration of antiretroviral treatment was much longer for group FT than for group NF $(p<0.0001)$. Very few individuals from the FT group had been in treatment for less than three years, and therefore, no meaningful comparisons could be made between FT and NF for individuals treated during similar periods of time.

HIV-1 Genetic Subtype - A total of $83(85,5 \%)$ samples could be successfully subtyped analyzing env C2V3 sequences and $82(84.5 \%)$ using PR and RT sequences. A total of 57 samples $(69.5 \%)$ showed concordance in subtyping determination using these genomic regions. Overall, the majority of the samples were classified as HIV-1 subtype B (80\%), followed by subtype F (14\%), BF recombinant forms $(5 \%)$. HIV-1 subtype C was identified in one patient $(1 \%)$. No preferential distribution of genetic HIV-1 subtypes could be observed in the different study groups.

Drug Resistance Mutations - In the treatment-naïve group (NT) various combinations of secondary (minor) protease mutations at codon positions L63P (23\%), M36I (18\%), A71V and V77I (9\% each) were observed. At baseline, the major mutation Y181C associated to NNRTI and the V118I and F77L secondary mutations associated to NRT inhibitors were observed in one patient each $(2,5 \%$ each).

The development of resistance-related mutations in genotyped samples from treated patients failing HAART (FT) showed that 70\% had resistance to NRTI, 55\% to the NNRTI and $45 \%$ to the protease inhibitors. Overall, a reduction of ARV susceptibility observed was around $40 \%$ to one class of drug, $20 \%$ to two classes of drugs and $30 \%$ against 3 classes of drugs. Only $10 \%$ of the genotyped FT samples showed complete susceptibility to all classes of ARV, and only $4 \%$ of the successful therapy group showed reduction of two classes: NRTI and NNRTI drugs. Presence of resistance-related mutations in genotyped samples from NF successfully treated patients showed that $10 \%$ of them had reduction of susceptibility to NRTI drugs and $15 \%$ to NNRTI antiretrovirals. No associated resistance mutations were observed simultaneously for both classes of ARV drugs.
V3-peptide Binding Antibodies - Frequency of reactivity and recognition titers were unexpectedly high for plasma from the FT group. As Fig. 1 shows, reactivity was lower for plasma from individuals undergoing successful therapy (group NF), and higher for FT individuals. However, statistical analyses showed significance for only some peptide recognition frequencies and reactivity titers. Thus, recognition of the reference gp41 immunodominant peptide (ID) was higher for FT than NF plasma $(p=0.0133)$, with higher titers $(p=0.0411)$. Frequencies were also higher for FT than NF for the Brazilian consensus sequence V3Bbr $(p=0.0001)$, and even higher than for the treatment-naïve control group NT $(p=0.0024)$. Differences in reactivity frequencies were highest for peptides corresponding to consensus sequences of HIV-1 subtypes prevalent in Rio de Janeiro (B, Bbr, F, Fbr), city of residence for all individuals participating in this study. Analyses of the frequency of peptide recognition by plasma from individuals infected with $\mathrm{B}$, Bbr or F HIV-1 showed that B and F plasma recognized V3B and V3F to a similar extent, but Bbr plasma showed a much more specific recognition of V3Bbr than V3B $(\mathrm{p}=0.0209)$, V3F $(\mathrm{p}=0.0038)$ or V3Fbr $(\mathrm{p}=$ $0.0005)$. This finding was also expressed by the higher titers of NT-Bbr plasma reactivity with V3Bbr in comparison to reactivity with $\mathrm{V} 3 \mathrm{~B}(\mathrm{p}=0.007), \mathrm{V} 3 \mathrm{~F}(\mathrm{p}=$ $0.0010)$ or $\mathrm{V} 3 \mathrm{Fbr}(\mathrm{p}<0.0001)$, with similar results being observed for FT-Bbr plasma, while the number of plasma in the NF-Bbr group was too low to permit comparison within the group. A higher titer of $\mathrm{F}$ plasma with the V3F peptide than V3Bbr or V3Fbr was again statistically significant only within the FT group. However, the number of plasma in each group was too small to permit definite conclusions to be drawn.

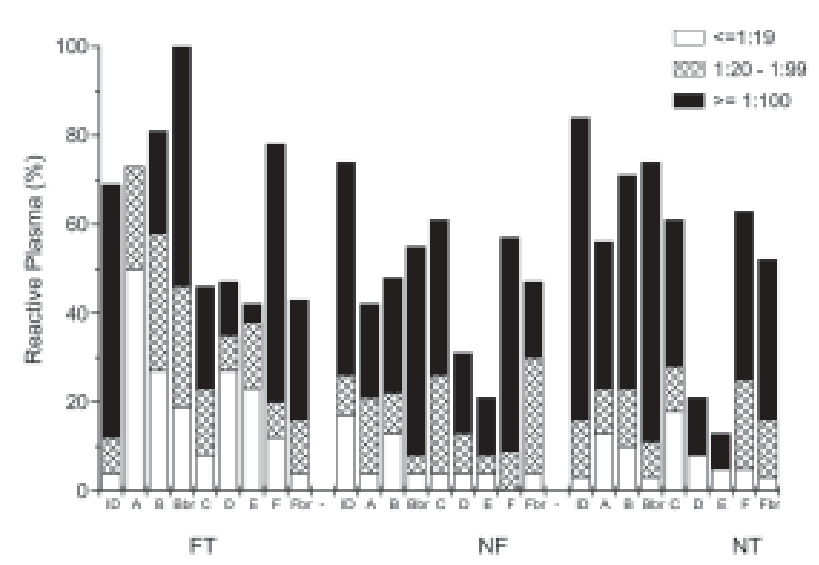

Fig. 1: Reactivity of plasma from individuals with failures of therapy (FT), from individuals in successful therapy with no failures (NF), and treatment naïve individuals with no antiretroviral therapy (NT) with peptides corresponding to consensus sequences of the gp41 immunodominant epitope ID and sequences covering the top of the V3 loop of HIV-1 of the consensus for subtype A (A), subtype B (B), the Bbr variant of subtype B (Bbr), subtype C (C), subtype D (D), subtype E (E), subtype F (F) and a Brazilian variant $\mathrm{Fbr}(\mathrm{Fbr})$. Highest reactive plasma dilutions were stratified into low $(\leq 1: 19)$, intermediate $(1: 20$ to $1: 99)$ and high titer $(\geq 1: 100)$. 
HIV-1 Neutralizing antibodies - A total of 5 HIV-1 isolates were used for the analysis, although not all plasma could be tested against all isolates due to limited amounts of primary HIV-1 isolate stocks available. Unfortunately, only isolates from individuals in the NT group could be obtained with the high titers needed for neutralizing antibody assays. Two primary isolates of the $\mathrm{F}$ subtype (FT15 and RJ21) and two isolates of the B subtype (FT89 and RJ20) were employed, as was the reference isolate HIV-1 IIIB (also subtype B, adapted to growth in lymphocytic cell lines although an expansion in human lymphocytes was employed in the assays).

Plasma from treated patents, successfully (NF group) or unsuccessfully (FT group), were less frequently efficient in neutralizing the different HIV-1 isolates, mostly in comparison to plasma from treatment naïve individuals (group NT), with statistically significant differences for neutralization of IIIB ( $90 \%$ neutralization levels, $\mathrm{p}=$ $0.0167)$. Mostly, however, statistically significant differences were observed at $50 \%$ levels of neutralization of viral input, and at lower plasma dilutions, where the effect of plasmatic antiretroviral drugs can have affected neutralization results.

Broadness of the response, defined as neutralizing more than half the isolates analyzed, indicated a more limited cross neutralization for individuals of the group of plasma from patients with undetectable viral load for at least one year. However, only a few plasma from the FT group showed capacity to neutralize $90 \%$ of the input of at least two of three primary HIV isolates tested $(22 \%)$, while the majority of the plasma from the NT group $(73 \%)$ did neutralize the majority of the HIV-1 isolates tested (NT $v_{s} \mathrm{FT}, \mathrm{p}=0.0166$; NT $v_{s} \mathrm{NF}, \mathrm{p}=0.0127$ ), even if only at lower plasma dilutions. Fig. 2 indicates the similarity in mean neutralization titers observed for FT and NF plasma, with no important differences or trends noticeable. No data is shown for neutralization of HIV-1 FT89 by FT plasma as too few plasma (only five) from individuals in actual therapy failure were used in the assay, as revealed by the decoding of the plasma samples carried out after the assays were finished, a precaution taken to diminish objective evaluation of the data.

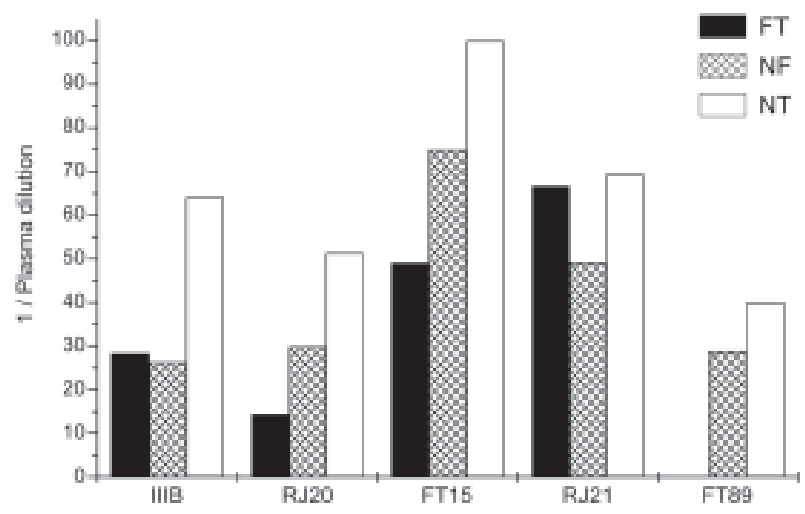

Fig. 2: HIV-1 neutralization by plasma from individuals in failure of therapy (FT) and successful therapy without failure (NF) in comparison to nontreated individuals (NT). Mean dilution of FT, NF and NT plasma achieving $90 \%$ neutralization of the HIV-1 isolates IIIB, RJ21, FT15, RJ20 and FT89.
Humoral immune response and viral load analyses Although identification of failure of therapy was based on high viremia peaks during treatment, for some of the patients viral loads (VL) were already quite low during sampling. Therefore, an analysis based on actual viral load during the last year before sampling allowed a division of the patients into two groups, one (group 1, Gr1) who had undetectable viral loads for at least one year before sampling (two to four VL determinations) and one (group 2, Gr2) with viral loads above 1,000 copies/ ml plasma in all evaluations during the last year before sampling. Fig. 3a shows an example of the higher recogni-
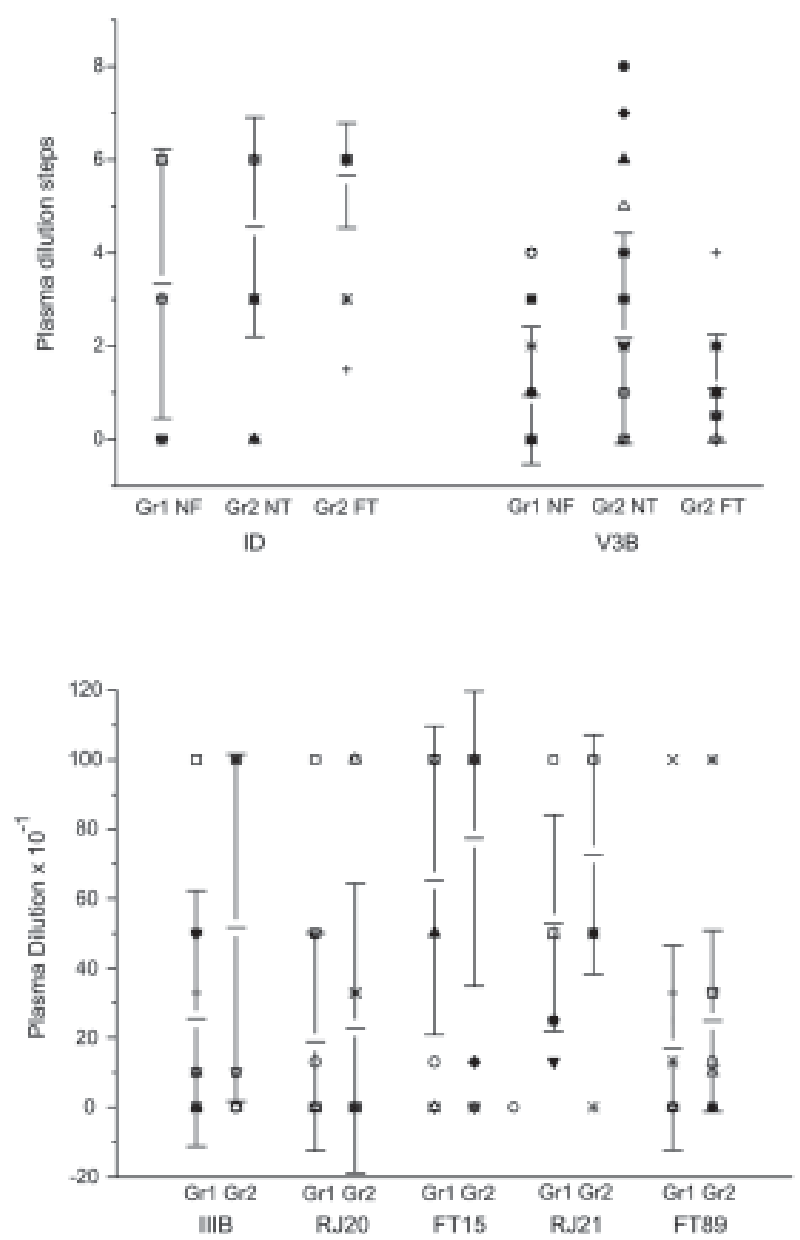

Fig. 3: Humoral immune response of plasma from individuals divided into group 1 ( $\mathrm{Gr} 1=$ individuals who had viral loads below the detection limit for at least one year before sample collection) and group $2(\mathrm{Gr} 2=$ individuals with viral loads above $1000 \mathrm{copies} / \mathrm{ml}$ in the two to four viral load quantifications made during one year before plasma collection). Gr1 NF= "no failure" = successfully treated individuals with undetectable viral loads for $\geq$ one year; Gr2 NT = therapy naïve individuals with viral loads above 1000 during the last year before sample collection; Gr2 FT = individuals with therapy failures and with viral loads above 1000 during the last year before sample collection. a: binding of antibodies to the synthetic gp41 immunodominant peptide (ID) and to the consensus V3B peptide; b: comparison of plasma dilutions needed for $90 \%$ neutralization of the HIV-1 isolates IIIB, RJ20, FT15, RJ21 and FT89. Mean values and standard deviations are indicated for each group of plasma, as well as outlier values. 
tion of synthetic peptides observed for plasma from individuals with detectable viral loads (group 2), although statistically significant only for the g41 ID peptide (Gr1 vs Gr2 FT, $\mathrm{p}=0.00252$; Gr 1 vs Gr2 NT, $\mathrm{p}=0.0252$ ). Similarly, neutralization titers in plasma from group 2 individuals were higher than those from group 1 , in neutralization of all 5 HIV-1 isolates tested (Fig. 3b). However, these differences had no statistical significance $(\mathrm{p}>0.05)$.

Anti-HIV antibodies vs CD4 lymphocyte counts and vs therapy failures - No relationship between number of CD4 T lymphocytes at sample collection and HIV-1 neutralization could be observed. Equally, no correlation between the nadir of CD4 cell concentration and HIV neutralization or reactivity of the plasma with synthetic V3 peptides could be observed, although a slight trend for higher HIV-1 neutralization titers (but not V3 peptide binding antibodies) was observed in individuals with a higher number of therapy failures $(p=0.09)$ (data not shown).

\section{DISCUSSION}

As several studies have shown a stimulation of the immune response by structured therapy interruptions or episodes of viral rebound or "blips" during successful HAART (Binley et al. 2000, Ioannides et al. 2000, Montefiori et al. 2001, Kimura et al. 2002, Wang et al. 2002, Sullivan et al. 2003, Karlson et al. 2004, Macias et al. 2005, Benito et al. 2006, Jacobson et al. 2006, Killian et al. 2006), it could be expected that a similar stimulation might occur during increases in viremia during therapy failure episodes, at least in individuals whose immunodeficiency is not advanced. This could be an explanation for the unexpected good health observed despite treatment failure in many patients (Brígido et al. 2004, Kovacs et al. 2005). The results presented indicate that individuals who have had episodes of therapy failure indeed show higher frequencies and titers of antibodies recognizing synthetic peptides corresponding to the immunodominant epitope in gp41 and peptides corresponding to consensus sequences of the V3 loop of some HIV-1 genetic subtypes. As expected, the main difference was observed for V3 peptides corresponding to HIV-1 genetic subtypes prevalent in the state of Rio de Janeiro, where all patients included resided. As in Rio de Janeiro the prevalent HIV-1 subtypes, B and F, both present the GPGR sequence at the top of the V3 loop, and the top of the less often recognized $\mathrm{V} 3$ peptides (A, $\mathrm{D}$ and $\mathrm{E})$ present the GPGQ sequence, this amino acid substitution may be of importance for the antibody response, as should be expected by the difference in charge and size of the residual groups of the amino acids arginine and glutamine. However, this does not explain the very high reactivity observed in FT plasma with the F peptide. Among 70 Brazilian subtype F sequenced env (most from the southeastern region of the country), approximately $34 \%$ display the GPGQ tetrapeptide at the top of the V3 loop, and the great majority of these, as well as the B, Bbr and Fbr V3 consensus sequences, have a great similarity in amino acid sequence of the $\mathrm{V} 3$ loop anterior arm (G Bello, personal communication). The similarity of humoral immune response between B and $\mathrm{F}$ subtype infected individuals could again be verified in the study presented, as differences between B and F plasma were very minor. Plasma from HIV-Bbr individuals, on the other hand, showed a significant preferential recognition of the homologous V3Bbr consensus. Unfortunately, no isolate identified as belonging to the Bbr variant of the B subtype could be produced in the titers and quantities necessary for neutralization assays, so the question of the susceptibility of Bbr primary isolates cannot be discussed in this study. However, plasma from HIV-Bbr infected individuals neutralized the B and F HIV-1 isolates tested to a similar extent as the $\mathrm{B}$ and $\mathrm{F}$ plasma identified.

No association between HIV-1 subtype and disease progression could be observed, however, the data reveal an elevated frequency of drug resistance mutations in the protease gene among drug-naïve individuals from Rio de Janeiro city. The profile of drug resistance mutations in the treated groups showed correlation with the therapy employed and no differences were observed between failure and responder groups. The presence of resistance associated mutations against different classes of ARV drugs supports the importance of resistance testing for guidance of therapy.

The neutralization analyses carried out indicate that the stimulation of NAb was not as obvious as that of the bAb: HIV-1 neutralization was quite low for the majority of the plasma analyzed, with few plasma neutralizing any of the isolates at dilutions above 1:50, considered to be a dilution where the drugs present in the plasma of the FT and NF individuals would not affect HIV-1 replication in the NAb assay. However, it is very difficult to be sure of this effect in neutralization assays, as of the 51 plasma tested only three were able to neutralize all isolates tested. The other 48 plasma were able to neutralize one or more isolates but showed no neutralization of at least one isolate tested, even at the 1:10 dilution, indicating a lack of interference of the antiretroviral drugs taken.

Agreeing with results obtained before (Bongertz et al. 2005), the F subtype primary HIV-1 isolates were significantly more susceptible to neutralization than the $\mathrm{B}$ subtype primary isolates, and even more susceptible than the T cell line adapted isolate HIV-1 IIIB.

Broadness of the neutralizing antibody response was highest for plasma from treatment naïve individuals, but very low in individuals who had failures of therapy, significantly lower than in individuals with successful therapy, although the time periods since HIV-1 diagnosis were highest for individuals in group FT, intermediate for those in group NF and shortest for those in the NT group. However, a trend to higher cross neutralization in individuals infected for a shorter period of time appeared to be more relevant than the actual viral loads determined in the year before sample collection, as suggested by the data from the plasma in the study by Kim et al. (2001).

Studies have shown that the CD4 T lymphocyte nadir is very important for reconstitution of cellular immune response during/ after HAART (Siddique et al. 2006). An analysis of nadir CD4 levels, however, showed no 
correlation between these values and the capacity for neutralizing the HIV-1 isolates included in this study.

A surprisingly high proportion of individuals with no detectable viral loads for the last year before sample collection showed a good humoral immune response, both in antibodies capable of binding V3 peptides and in antibodies effective in controlling heterologous HIV-1 replication in vitro. Although seroreversion appears to be rare even after years of successful antiviral therapy, the gradual decrease of specific anti-HIV-1 antibodies is a well known occurrence in HAART patients (Béniguel et al. 2004, Amor et al. 2006, Cornelissen et al. 2006). Several hypotheses have been raised to explain the maintenance of a humoral anti-HIV-1 immune response after years of apparent absence of HIV-1 antigenic stimulation, including maintenance of HIV-1 antigens associated to follicular dendritic cells in patients with undetectable viremia (Popovic et al. 2005).

Based on the results presented here, therapy failure, similar to rebounds in viremia, will lead to a stimulation of anti-HIV-1 antibodies, able to recognize specific HIV1 epitopes but apparently unable to effectively control HIV-1 replication, as HIV-1 neutralization does not appear to be significantly stimulated by the increase in antigen availability. However, the lack of autologous neutralization analyses of this study prevents meaningful conclusions upon the effectiveness or clinical benefit for the individual patients. Although no broader neutralization response was seen in individuals of the FT group, it seems possible that years of HAART, while not completely inhibiting viral replication, may at least in part control the highly infectious and "fit" viral subpopulation, and, possibly, give rise to "antigenic debris". These debris (Parren et al. 1997) may be responsible for the induction of a specific immune response observed by binding to synthetic peptides, but are unable to induce production of antibodies capable of interfering with cellular infection by HIV-1.

\section{ACKNOWLEDGEMENTS}

To the patients for participating in this study, to the donors and the Haematology Service of the Clementino Fraga Filho Hospital, UFRJ, for donation of surplus buffy coats. To Dr EvaMaria Fenyoe for donation of the HIV-1 IIIB reference isolate. The technical assistance of Luciano S Amaral, Vanessa Martins and Giselle Furtado Borges is gratefully acknowledged.

\section{REFERENCES}

Amor A, Toro C, Jimenez V, Simon A, Ramos B, Soriano V 2006. Seroreversion of HIV antibodies in patients with prolonged suppression of viraemia under HAART. AIDS 20: 1460-1462.

Bailey JR, Lassen KG, Yang HC, Quinn TC, Ray SC, Blankson JN, Siliciano RF 2006. Neutralizing antibodies do not mediate suppression of human immunodeficiency virus type 1 in elite suppressors or selection of plasma virus variants in patients on highly active antiretroviral therapy. J Virol 80:4758-4770.

Béniguel L, Begaud E, Peruchon S, Cognasse F, Gabrie P, Marovich M, Lucht F, Genin C, Garraud O 2004. Isotype profiles of anti-gp160 antibodies from HIV-infected patients in plasma and culture supernatants. Immunol Lett 93: 57-62.
Benito JM, Lopez M, Ballesteros C, Lozano S, Capa L, Barreiro P, Sempere J, Gonzalez-Lahoz J, Soriano V 2006. Immunological and Virological Effects of Structured Treatment Interruptions following Exposure to Hydroxyurea Plus Didanosine. AIDS Res Hum Retroviruses 22: 734-743.

Binley JM, Trkola A, Ketas T, Schiller D, Clas B, Little S, Richman D, Hurley A, Markowitz M, Moore JP 2000. The effect of highly active antiretroviral therapy on binding and neutralizing antibody responses to human immunodeficiency virus type 1 infection. J Infect Dis 182: 945-949.

Bongertz V, Bou-Habib DC, Brigido LFM, Caseiro M, CoutoFernandez JC, Ferreira PC, Freitas CO, Galvão-Castro B, Greco D, Guimarães ML, Linhares-de-Carvalho MI, Morgado MG, Oliveira CAF, Osmanov S, Ramos CA, Rossini M, Sabino E, Tanuri A, Ueda M 2000. HIV-1 diversity in Brazil: genetic, biological and immunological characterization of HIV1 strains in three potential HIV vaccine evaluation sites. Brazilian Network for HIV Isolation and Characterization. $J$ Acquir Immune Def Syndr 23: 184-193.

Bongertz V, Costa CI, Veloso VG, Grinsztejn B, Filho EC, Calvet G, Pilotto JH 2001. Vertical HIV-1 transmission: importance of neutralizing antibody titer and specificity. Scand J Immunol 53: 1-9.

Bongertz V, Ouverney EP, Teixeira SLM, C Silva-de-Jesus, MA Hacker, MG Morgado, FI Bastos, The Brazilian Network for HIV Isolation and Characterization 2003. Higher antiHIV-1 antibody titers in injection drug users compared to sexually infected individuals. Mem Inst Oswaldo Cruz 98: 209-212.

Bongertz V, Teixeira SL, Grinztejn B, Pilotto JH, Veloso VG, Morgado MG, Bastos FI, Ouverney EP 2005. Human immunodeficiency virus type 1 neutralization by plasma from B or F genotype infected individuals. Mem Inst Oswaldo Cruz 100: 85-89.

Brígido L, Rodrigues R, Casseb J, Custodio RM, Fonseca LA, Sanchez M, Duarte AJ 2004. CD4+ T-cell recovery and clinical outcome in HIV-1-infected patients exposed to multiple antiretroviral regimens: partial control of viremia is associated with favorable outcome. AIDS Patient Care STDS 18:189-198.

Bucy RP, Kilby JM 2001. Perspectives on inducing efficient immune control of HIV-1 replication - a new goal for HIV therapeutics? AIDS Suppl 2: S36-S42.

Casseb J, Katzenstein D, Winters M, Brigido LF, Duarte AJ, Hendry RM 2002. Serotyping HIV-1 with V3 peptides: detection of high avidity antibodies presenting clade-specific reactivity. Braz J Med Biol Res 35: 369-375.

Chehimi J, Azzoni L, Farabaugh M, Creer SA, Tomescu C, Hancock A, Mackiewicz A, D’Alessandro L, Ghanekar S, Foulkes AS, Mounzer K, Kostman J, Montaner LJ 2007. Baseline viral load and immune activation determine the extent of reconstitution of innate immune effectors in HIV-1infected subjects undergoing antiretroviral treatment. $J$ Immunol 179: 2642-2650.

Cheonis N 2005. Immune reconstitution syndrome. BETA 17:12-15.

Cornelissen M, Jurriaans S, Prins JM, Bakker M, Van der Kuyl AC 2006. Absence of seroreversion in 80 HAART-treated HIV-1 seropositive patients with at least five-years undetectable plasma HIV-1 viral load. AIDS Res Ther 3: 3 .

Dalod M, Harzic M, Pellegrin I, Dumon B, Hoen B, Sereni D, 
Deschemin JC, Levy JP, Venet A, Gomard E 1998. Evolution of cytotoxic T lymphocyte responses to human immunodeficiency virus type 1 in patients with symptomatic primary infection receiving antiretroviral triple therapy. $J$ Infect Dis 178: 61-69.

David D, Pires R, Treilhou MP, Dupont B, Joussemet M, Pialoux G, Theze J, Bouvet JP 1999. Downregulation of the expression of the main immunoglobulin $\mathrm{V}(\mathrm{H})$ family in HIV-infected patients: modulation by triple combination therapy. AIDS Res Hum Retroviruses 15: 315-316.

Devito C, Hejdeman B, Albert J, Broliden K, Hinkula J 2006. Antiretroviral therapy does not induce HIV type 1-specific neutralizing activity against autologous HIV type 1 isolates. AIDS Res Hum Retroviruses 22: 908-911.

Dreyer K, Kallas EG, Planelles V, Montefiori D, McDermott MP, Hasan MS, Evans TG 1999. Primary isolate neutralization by HIV type 1-infected patient sera in the era of highly active antiretroviral therapy. AIDS Res Hum Retroviruses 15: 1563-1571.

Eyer-Silva, WA, Morgado, MG 2006. Molecular Epidemiology of HIV-1 Infection in a Small Brazilian County: Usefulness of Envelope and Polymerase Sequences to Epidemiologic Studies. J Acquir Immune Def Syndr 41: 664-670.

Fournier AM, Baillat V, Alix-Panabieres C, Fondere JM, Merle C, Segondy M, Huguet MF, Reynes J, Vendrell JP 2002. Dynamics of spontaneous HIV-1 specific and non-specific B-cell responses in patients receiving antiretroviral therapy. AIDS 16: 1755-1760.

Guimarães ML, dos Santos Moreira A, Loureiro R, Galvão-Castro B, Morgado MG 2002. High frequency of recombinant genomes in HIV type 1 samples from Brazilian southeastern and southern regions. AIDS Res Hum Retroviruses 18: 1261-1269.

Ioannidis JP, Havlir DV, Tebas P, Hirsch MS, Collier AC, Richman DD 2000. Dynamics of HIV-1 viral load rebound among patients with previous suppression of viral replication. AIDS 14: 1481-1488.

Jacobson JM, Pat Bucy R, Spritzler J, Saag MS, Eron JJ, Jr, Coombs RW, Wang R, Fox L, Johnson VA, Cu-Uvin S, Cohn SE, Mildvan D, O'neill D, Janik J, Purdue L, O'connor DK, Vita CD, Frank I, National Institute of Allergy and Infectious Diseases-AIDS Clinical Trials Group 5068 Protocol Team 2006. Evidence That Intermittent Structured Treatment Interruption, but Not Immunization with ALVAC-HIV vCP1452, Promotes Host Control of HIV Replication: The Results of AIDS Clinical Trials Group 5068. J Infect Dis 194: 623-632.

Karlsson AC, Younger SR, Martin JN, Grossman Z, Sinclair E, Hunt PW, Hagos E, Nixon DF, Deeks SG 2004. Immunologic and virologic evolution during periods of intermittent and persistent low-level viremia. AIDS 18: 981-989.

Killian MS, Norris PJ, Rawal BD, Lebedeva M, Hecht FM, Levy JA, Busch MP 2006. The Effects of Early Antiretroviral Therapy and Its Discontinuation on the HIV-Specific Antibody Response. AIDS Res Hum Retroviruses 22: 640-647.

Kim JH, Mascola JR, Ratto-Kim S, VanCott TC, Loomis-Price L, Cox JH, Michael NL, Jagodzinski L, Hawkes C, Mayers D, Gilliam BL, Birx DC, Robb ML 2001. Selective increases in HIV-specific neutralizing antibody and partial reconstitution of cellular immune responses during prolonged, successful drug therapy of HIV infection. AIDS Res Hum Retroviruses 17: 1021-1034.
Kimura T, Yoshimura K, Nishihara K, Maeda Y, Matsumi S, Koito A, Matsushita S 2002. Reconstitution of spontaneous neutralizing antibody response against autologous human immunodeficiency virus during highly active antiretroviral therapy. $J$ Infect Dis 185: 53-60.

Kovacs A, Montepiedra G, Carey V, Pahwa S, Weinberg A, Frenkel L, Capparelli E, Mofenson L, Smith E, McIntosh K, Burchett SK, Pediatric AIDS Clinical Trials Group 366 Study Team. 2005. Immune reconstitution after receipt of highly active antiretroviral therapy in children with advanced or progressive HIV disease and complete or partial viral load response. J Infect Dis 192: 296-302.

Kumar M, Keller B, Makalou N, Sutton RE 2001. Systematic determination of the packaging limit of lentiviral vectors. Hum Gene Ther 12:1893-1905.

Macias J, Palomares JC, Mira JA, Torres MJ, Garcia-Garcia JA, Rodriquez JM, Vergera S, Pineda JA 2005.Transient rebounds of HIV plasma viremia are associated with the emergence of drug resistance mutations in patients on highly active antiretroviral therapy. $J$ Infect 51:195-200.

Manzardo C, Zaccarelli M, Aguero F, Antinori A, Miro JM 2007. Optimal timing and best antiretroviral regimen in treatmentnaive HIV-infected individuals with advanced disease. $J$ Acquir Immune Def Syndr 46 (Suppl I): S9-18.

Mocroft A, Ledergerber B, Katlama C, Kirk O, Reiss P, d'Arminio Monforte A, Knysz B, Dietrich M, Phillips AN, Lundgren JD, EuroSIDA study group 2003. Decline in the AIDS and death rates in the EuroSIDA study: an observational study. Lancet 362: 22-29.

Montefiori DC, Hill TS, Vo HT, Walker BD, Rosenberg ES 2001. Neutralizing antibodies associated with viremia control in a subset of individuals after treatment of acute human immunodeficiency virus type 1 infection. J Virol 75: 10200-10207.

Morris L, Binley JM, Clas BA, Bonhoeffer S, Astill TP, Kost R, Hurley A, Cao Y, Markowitz M, Ho DD, Moore JP 1998. HIV-1 antigen-specific and -nonspecific B cell responses are sensitive to combination antiretroviral therapy. J Exp Med 188: 233-245.

Morris MK, Katzenstein DA, Israelski D, Zolopa A, Hendry RM, Hanson CV 2001. Characterization of the HIV-1 specific humoral immune response during highly active antiretroviral therapy (HAART). J Acquir Immune Def Syndr 28: 405-415.

Parren PW, Burton DR, Sattentau QJ 1997. HIV-1 antibodydebris or virion? Nat Med 3: 366-367.

Popovic M, Tenner-Racz K, Pelser C, Stellbrink HJ, van Lunzen J, Lewis G, Kalyanaraman VS, Gallo RC, Racz P 2005. Persistence of HIV-1 structural proteins and glycoproteins in lymph nodes of patients under highly active antiretroviral therapy. Proc Natl Acad Sci USA 102: 14807-14812.

Siddique MA, Hartman KE, Dragileva E, Dondero M, Gebretsadik T, Shintani A, Peiperl L, Valentine F, Kalams SA 2006. Low CD4+ T Cell Nadir Is an Independent Predictor of Lower HIV-Specific Immune Responses in Chronically HIV-1-Infected Subjects Receiving Highly Active Antiretroviral Therapy. J Infect Dis 194: 661-665.

Soares MA, Brindeiro RM, Tanuri A 2004. Primary HIV-1 drug resistance in Brazil. AIDS 18 (Suppl III): S9-S13.

Sullivan AK, Burton CT, Nelson MR, Moyle G, Mandalia S, Gotch FM, Gazzard BG, Imami N 2003. Restoration of human im- 
munodeficiency virus-1-specific responses in patients changing from protease to non-nucleoside reverse transcriptase inhibitorbased antiretroviral therapy. Scand J Immuno 57: 600-607.

UK Collaborative Group on HIV Drug Resistance, UK Collaborative Cohort Study, UK Register of HIV Seroconverters 2007. Evidence of a decline in transmitted HIV drug resistance in the United Kingdom. AIDS 21: 1035-1039.

Vermund SH 2006. Millions of life-years saved with potent antiretroviral drugs in the United States: a celebration, with challenges. J Infect Dis 194: 1-5.
Wang FX, Kimura T, Nishihara K, Yoshimura K, Koito A, Matsushita S 2002. Emergence of autologous neutralizationresistant variants from preexisting human immunodeficiency virus (HIV) quasi species during virus rebound in HIV type 1infected patients undergoing highly active antiretroviral therapy. J Infect Dis 185: 608-617. Erratum in: J Infect Dis 186: 145.

WHO-UNAIDS HIV-1 Vaccine Initiative, Initiative for Vaccine Research, Health Technology and Pharmaceutical, World Health Organization 2002. WHO-UNAIDS Guidelines for Standard HIV Isolation and Characterization Procedures. $2^{\text {nd }}$ ed., p 40-57. 